\title{
BMJ Open Patients' experiences of using an e- Health tool for self-management support after prostate cancer surgery: a deductive interview study explained through the FITT framework
}

To cite: Nilsson L, Hellström A, Wennerberg C, et al. Patients' experiences of using an eHealth tool for self-management support after prostate cancer surgery: a deductive interview study explained through the FITT framework. BMJ Open 2020;10:e035024. doi:10.1136/ bmjopen-2019-035024

- Prepublication history for this paper is available online. To view these files, please visit the journal online (http://dx.doi. org/10.1136/bmjopen-2019035024).

Received 19 0ctober 2019

Revised 07 April 2020

Accepted 29 May 2020

Check for updates

(c) Author(s) (or their employer(s)) 2020. Re-use permitted under CC BY-NC. No commercial re-use. See rights and permissions. Published by BMJ.

${ }^{1}$ eHealth Institute, Linnaeus University, Kalmar, Sweden ${ }^{2}$ Health and Caring Sciences, Linnaeus University, Kalmar,

Sweden

${ }^{3}$ Department of Surgery, Kalmar County Hospital, Kalmar,

Sweden

${ }^{4}$ Learning Informatics

Managment and Ethics,

Karolinska Institutet, Stockholm, Sweden

Correspondence to Professor Mirjam Ekstedt; mirjam.ekstedt@Inu.se

\section{ABSTRACT}

Objective To evaluate patients' experiences of using a web-based application, especially its usability as support for self-care activities after prostate cancer surgery. Design A deductive content analysis was used, stemming from the Fit between Individuals, Task and Technology (FITT) framework

Setting One surgical department in south of Sweden between October 2015 and April 2016 and between September 2017 and July 2018.

Participants Fifteen men who had undergone radical prostatectomy for prostate cancer.

Results By organising data in accordance with the FITT model, three main categories with ten subcategories were identified. Patients gave feedback on functions that suited them and their needs, as well as potential adjustments and improvements. Patients experienced that ePATH gave them easy access to reliable information regarding their rehabilitation. Directed information about ePATH at enrolment was seen as important. ePATH was perceived to have a logical structure that was easy to follow. However, when the structure was unclear, patients became less motivated to use a function.

Conclusions Patients experienced ePATH as satisfactorily user-friendly and useful as a complementary selfmanagement support after prostate cancer surgery, especially when the information and tasks were tailored to their preferences and the system design features supported individual autonomy.

\section{BACKGROUND}

In 2015, a standardised care pathway (SCP) for prostate cancer was launched by the Swedish government. ${ }^{1}$ As part of the SCP, patients are assigned a contact nurse, whose task is to facilitate access to care. The nurse provides information and education related to the disease, treatment and self-care activities, supports patients and relatives, coordinates contacts within healthcare and assesses and monitors the patient's symptoms. ${ }^{2}$ However, a recent study in men diagnosed

\section{Strengths and limitations of this study}

- The usability testing was conducted at one surgical department in a medium-sized hospital in the south of Sweden.

- A sample of 15 men could be considered too small to provide reliable data, but even smaller sample sizes are quite common and can be meaningful as they cover a variety of unique experiences.

- The voluntary nature of patient participation may have led to our results not being transferable to other patients with prostate cancer or to men and women with other cancer diagnoses.

- The usability testing was not video recorded and some difficulties in using functionalities may have passed unnoticed.

- We used the Fit between Individuals, Task and Technology framework to describe and analyse the current fit between individual, task and technology, to identify how to improve the interaction between those aspects in ePATH.

with prostate cancer showed that the patients felt that they were in a vacuum and constantly waiting for information, both before diagnosis and during and after treatment. A need for more personalised support to patients and their families, especially in rehabilitation after treatment, was suggested. ${ }^{3}$ Shortcomings in the rehabilitation after surviving cancer have been found in other studies as well, and improved follow-up and effective interventions have been called for. ${ }^{4}$ e-Health applications have the potential to provide support to everyone, everywhere, and to facilitate contact with healthcare services when it is most convenient for the user.

There are several studies describing development and evaluation of e-Health tools in prostate cancer care. ${ }^{5-8}$ However, the applications have different focus and 
some lack theoretical basis for their content or interface. ${ }^{689}$ Golsteijn $e t a l^{7}$ focus solely on physical activities, while Davis et $a l^{5}{ }^{5}$ Frankland et $a l^{6}$ and Bartlett $e t$ $a l^{9}$ focus on information and guidance to follow up in accordance with the standardised care plan. Several of the applications apply to cancer illness in general or to multiple diagnoses, ${ }^{5-9}$ which makes tailoring of the content more difficult. Ruland $e t a l^{8}$ investigated several important components, such as long-term follow-up, interactive forums with contact nurses and other patients, and support and monitoring of symptoms. This is the tool that most resembles the tool evaluated in this study with respect to multimodality and patient-driven use. Frankland $e t a l^{6}$ focused mainly on costs and differences between traditional appointment-based follow-up care and an online-supported self-management and follow-up care programme. Although attention to e-Health applications has increased in cancer care, few studies focus on e-Health applications as person-centred rehabilitation support in the aftermath of prostate cancer treatment. ${ }^{10}$ In response to this scarcity, we designed a web platform, based on postoperative self-management needs that have been considered essential by patients in past studies. ${ }^{3}$ It has a theoretical base, emphasising autonomy, competence and relatedness, and guides the patient up to 12 months after treatment. ${ }^{11}$

Thus, the aim of the current study was to evaluate patients' experiences of using a web-based application, especially its usability as support for self-care activities after prostate cancer surgery.

\section{METHODS}

\section{Design and theoretical framework}

The study consisted of usability testing of ePATH using a think-aloud technique and semistructured interviews. The goal was to identify design and functionality issues along with usability problems in using an e-Health application as support for self-care activities after prostate cancer surgery. Usability can be improved only with an understanding of the tasks that patients need to perform in the course of their 'self-management and rehabilitation work'. ${ }^{12}$ We, therefore, approached usability from the patients' perspective through the lens of the Fit between Individuals, Task and Technology (FITT) framework, to determine the Fit between Individual, Task and Technology. This framework was developed by Ammenwerth et $a l^{13}$ based on the idea that the adoption of e-Health support depends on the fit between user and technology, and between task and technology. This means that the fit in the interaction between the attributes is more important than the individuals attributes themselves, and this must also be considered. ${ }^{13}$ The assumption is that the attributes of the individual, the task and the technology affect the fit between them, which in turn will have an influence on the utilisation and adoption of the e-Health support (figure 1). In this study, the FITT framework is used to describe and analyse the current fit between

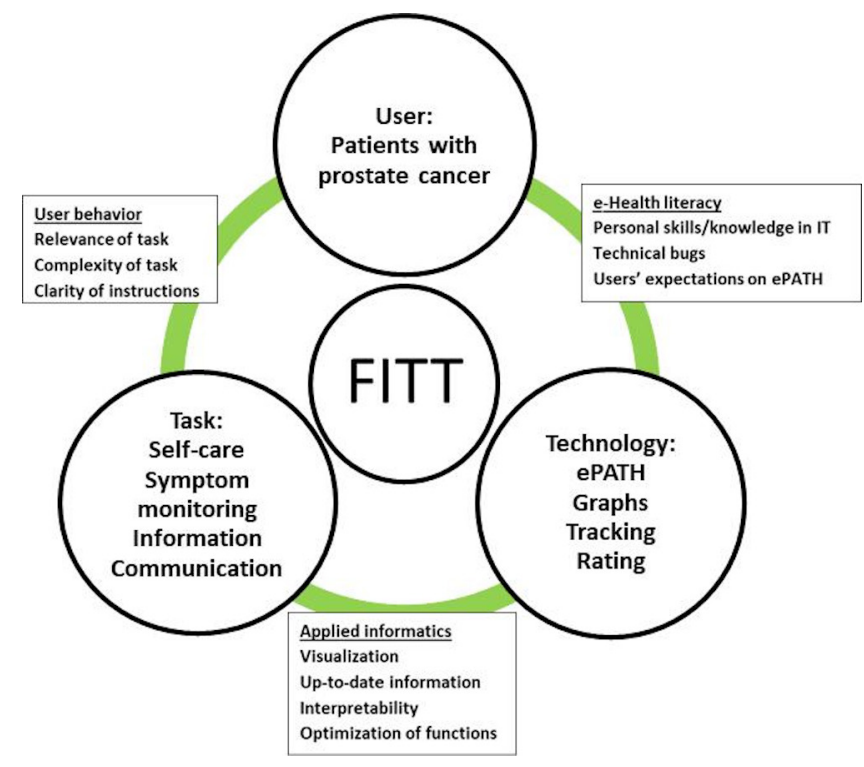

Figure 1 The FITT (fit between individuals, task and technology) framework, applied to usability testing of ePATH in prostate cancer patients.

individual, task and technology, which can be used to identify how to improve the interaction between those aspects in ePATH.

\section{The web-based application}

ePATH is an interactive e-Health application that is accessible through computer, smartphone or tablet devices, using secure authentication. The content and functionality of ePATH was developed with end users, by applying user-centred design. ${ }^{14-16}$ ePATH is based on five content areas that have been considered to be essential for posthospital self-management of care and rehabilitation. ${ }^{3} 1718$ Furthermore, ePATH is conceptually based on self-determination theory (SDT), ${ }^{11}$ with the assumption that intrinsic motivation, the 'drive' to sustain performance of self-care activities at home, is promoted by patient autonomy, competence and relatedness. Social contexts that support fulfilment of these basic psychological needs also facilitate more autonomous functioning. ${ }^{11} 19$ ePATH enhances patient autonomy by providing access to communication and interaction with healthcare professionals. Also, ePATH provides access to tailored information and self-care activities in partnership with the contact nurse. The patient can track self-care activities (e.g. physical activity and pelvic floor muscle training) and monitor symptoms (e.g. urinary leakage). The registrations are displayed numerically as tables or in graphs, and can be followed over time, providing feedback to enhance motivation. Important dates can be added to the calendar and there is also a private diary function. Further, there is a messaging function, allowing the patient to send text-based messages to his contact nurse, and the contact nurse has the possibility to respond or send supportive messages to the patient. ${ }^{20}$ 


\section{Patient and public involvement}

User-centred design approach was used as it provides a variety of methods (e.g. interviews, think-aloud techniques) to allow end users to influence how the product takes shape. ${ }^{131416}$ Patients who had undergone prostate cancer surgery were involved in the design and development of the ePATH platform by sharing their experiences, preferences and needs, which informed the content and functionality of ePATH. Patients were not involved in designing the research study, nor with service development with the entire ecosystem, but only the e-Health service. The results have been disseminated to the study participants and patient associations.

\section{Procedures}

The usability testing was conducted at the surgical department of a medium-sized hospital in the south of Sweden, to provide users (patients and contact nurses) with a system with a real look and feel and to determine the acceptance of the e-Health application and its features in everyday work. The contact nurses got an introduction to ePATH from the research team, during which they were able to test how to log in using their staff identification security card, and learnt how to introduce patients to ePATH, send messages and provide information. A user manual was developed as support for staff during the usability study. Eligible patients were informed about the study by their contact nurse in connection with their diagnosis (verbal and written information). Those who agreed to participate got an introduction to ePATH from the contact nurse. A written tutorial for patients was handed out, describing the full range of functions offered by ePATH. When the patients had been using ePATH for about 4 weeks, a researcher (CW) contacted each of them, asking for an interview to discuss their experiences of using the e-Health tool.

\section{Participants and setting}

Fifteen men, aged 56-72 years, diagnosed with prostate cancer and treated with radical prostatectomy, were invited to use ePATH postsurgery, and then participate in a follow-up interview. Inclusion criteria were to have access to a computer or tablet and a mobile BankID for secure login. All 15 men agreed to participate in the study and sampling ended after 15 usability tests and interviews, as no substantial new information was retrieved. Usability testing and interviews were made in two rounds, October 2015 to April 2016 and September 2017 to July 2018.

\section{Data collection methods}

After the introduction to ePATH from the contact nurse, patients who agreed to participate in the study were invited to test ePATH for 4 weeks postsurgery. During these weeks, they were encouraged to try out and use functions in ePATH. To set the date and time for interviews after the usability tests, one of the researchers (CW) contacted the patients who had agreed to participate in the study. Interviews were conducted in a conference room at the hospital by pairs of researchers (KS and OF, LN and CW). The usability testing started with a think-aloud session, followed by semistructured interviews loosely based on items in the modified System Usability Scale. ${ }^{21}$ The usability test sessions lasted from 11 to $57 \mathrm{~min}$ (mean=27). When showing the researchers how ePATH was used, users thought aloud while signing in, performing various activities within ePATH, and using various functions of ePATH. The think-aloud was guided by the same areas of function and content as described in the ePATH instructions. During the session, users reflected on how ePATH could be improved, that is, functions, communication and visualisation of patient data. During the interviews, probing questions were used to encourage the participants to elaborate on situations which were problematic or where they had concerns and to discuss possible causes or suggestions for improvements. All interviews were recorded and transcribed verbatim. In order to increase credibility, all quotations are presented with the quoted participant numbered.

All participants gave written informed consent to participate in the study. ${ }^{22}$

\section{Analysis of the interviews}

The transcribed interviews were analysed with content analysis using a deductive approach. ${ }^{23}$ As a first step, four authors (LN, CW, ME and KS) read all transcripts to obtain an understanding of the whole. The transcripts were then coded separately. In the next step, all authors discussed the interpretation of the data and compared the coding. A structured categorisation matrix was then developed based on the FITT framework, ${ }^{13}$ to sort data into the predefined categories (figure 1). The structure for figure 1 was made by one author $(\mathrm{AH})$. The relations between patients, ePATH and its content were used as an outline for the understanding of what influenced patients' utilisation of ePATH. Subcategories and main categories were discussed by all the authors and grouped based on how they were linked together. To increase trustworthiness, discussions in the research group continued until no inconsistencies existed and a shared understanding was reached.

\section{RESULTS}

Organising data in accordance with the FITT model, ten subcategories were identified and sorted into the three main categories. The latter captured the fit between patient, technology and task (figure 2).

\section{Turning input into output: task engagement and completion}

Patients found the opportunity to get trustworthy information about their diagnosis through the e-Health tool valuable. Patients reported that online searches for information about their illness sometimes took them to sites that did not provide reliable information. They felt that ePATH helped them to easily get information they could trust. 


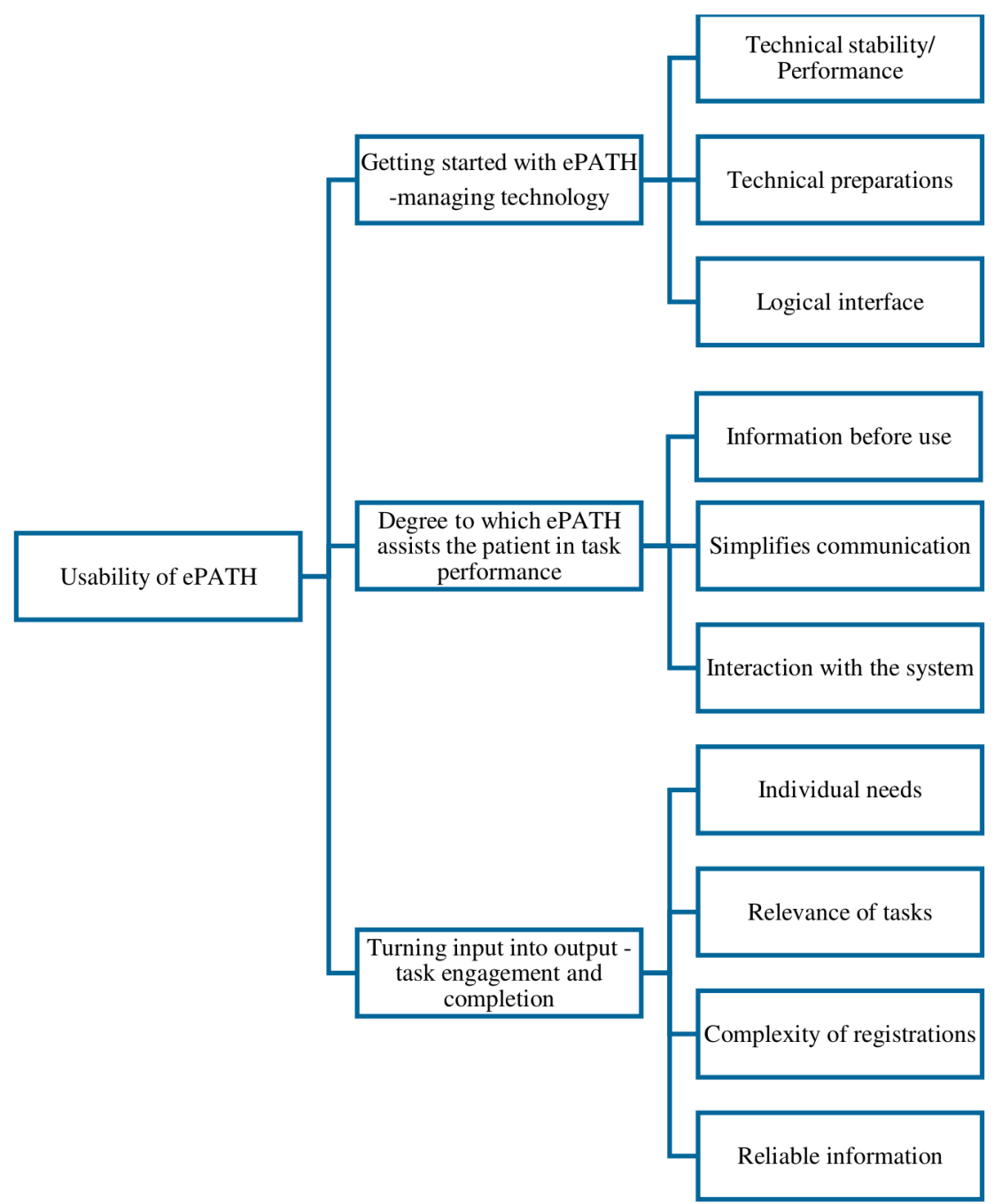

Figure 2 Usability of ePATH described through three main categories and ten subcategories.

Otherwise you end up sitting there, going online and checking around. There are sites that you can look at, but sometimes you get disinformation. (P 1)

Some of the patients found the self-care activity function useful for registering their self-care activities and symptoms, while others felt no need for such registration. The function could enhance or facilitate the understanding of which activities were most important and how often they should be done. The participants had different experiences of the usefulness of frequent registrations. Some felt that they lost motivation to register symptoms on a regular basis, when the symptoms changed little over time. They suggested that symptoms should be reported only when changes occurred. One patient stated that many things were going on in parallel during this time of life and found reminders unnecessary.

The motivation for and the feeling in relation to the rehabilitation process varied from day to day. Some patients wanted clear goals to focus on, while others did not have that need. The goals could awaken a competitive instinct that inspired more activity.
Yeah, but there's so much else. You need to know that it ... that this ... that how you feel and your general health and so on, it's a rollercoaster in this kind of situation. (P 8)

Patients had differing needs for the functions in the system and used those that suited them and their needs. The need for individual information directed at a specific patient was highlighted. Overly generic information was not perceived as useful; instead, the patients wanted ePATH to be tailored based on personal needs and requests. Patients wanted to cease with registration of selfcare activities and symptoms when such registration felt redundant. Instead, they wanted the possibility to register other self-care activities of importance for their rehabilitation, such as sleep patterns. Reminders to perform self-care activities were set to specific hours, but patients often performed them in connection with other daily activities. Patients emphasised that it was enough to get information about what to do and how many times a day. Psychoeducation about the importance of the activity was described as imperative. If patients did not understand why a task was recommended or how the self-care activity 
was assumed to affect their symptoms, they became less motivated to follow the instructions and register their activities in ePATH.

\section{So I use what I feel I need at the time. (P 6)}

Patients experienced benefits from the messaging function in ePATH. The possibility to write a message whenever it suited them and not have to adjust their life based on office hours was appreciated. It simplified contact with healthcare and created a sense of security. They described past difficulties with getting in contact with healthcare professionals and the frustration this had caused.

I think that's good. It is easier than that you have to ... call and wait on hold or ... Going in here and writing a message to the contact nurses, for instance, and maybe you'll get a response the very same day. That's much better than that I have to sit on the phone and ... on hold and maybe I won't reach them anyway. (P 11)

The need for clarity about the functions and parts in ePATH was highlighted. Patients had various suggestions on how the technical usability could be improved, such as creating an application you could use on smartphones and using notifications as reminders.

\section{Getting started with ePATH: managing technology}

Computers or tablets were used to access ePATH. The headings provided clear guidance on where different functions could be found. The tool was perceived to have a logical structure that was easy to follow. The interface and colour settings in the tool were considered to be good.

That layout is clear and distinct. It is not complicated. (P 15)

One patient had hoped to read his medical journal through the system and was disappointed when that was not possible. Connections between different systems or links were seen as a good function and could be a way to improve functionality. One example was to supply recommendations for podcasts that were suitable in the situation or links to patient information on prostate cancer.

Patients believed that the paperless society was the future and that e-Health tools like ePATH were a good way to get access to the information that you previously got on paper. According to the patients, ePATH gave access to the information wherever they had internet access, and there was no need to keep track of papers or phone numbers anymore, as you had the option to get in touch through ePATH.

\section{Degree to which ePATH assists the patient in task performance}

Clear information about what ePATH entailed was important at enrolment. In circumstances when information was not clear, the results were disappointment and lack of motivation for usage.
Patients encountered bugs and deficiencies, which confused some of them and made them question their digital literacy and skills. Others were certain that the shortcomings that occurred were due to technical problems.

No, but there's no message box, I can tell you that right away, because then I would have looked there, but there were no headings. (P 6)

And then I discovered that the registration here ... Sometimes it disappears. I've saved it and then it's gone. (P 2)

\section{DISCUSSION}

This study aimed to evaluate patients' experiences of using a web-based application, especially its usability as support for self-care activities after prostate cancer surgery.

\section{Fit between the individual and the technology}

The patients' impressions of using an e-Health tool were positive, in general. An important aspect in using ePATH was that the design and the interface were logical and clear that the view was up-to-date when they opened the application and that outdated activities or registrations were hidden. Provision of diagnosis-specific information, a bidirectional communication feature and the possibility to personalise timing and features of self-care activities based on preferences and needs were especially appreciated and motivating. Similarly, Lentferink $e t a t^{24}$ found that patients described usability as the ability to personalise and set the technical features of an application, such as timing of messages, layout or password protection. Personalisation of an application also included having an influence over its contents and the possibility to observe patterns or trends in personalised data. The patients were also willing to put more effort into registration of data if it added value for them. Performance of self-tracking was perceived as increasing awareness; however, registration of data seemingly irrelevant for the patient could be demotivating. ${ }^{245}$ The possibility of setting one's own goals and breaking these down to more manageable subgoals, has been found to be important from a user perspective ${ }^{26}$ and could add value to the content.

A good fit between the patient and technology, with a focus on personalisation and autonomous functioning, as shown in this study, may support patient engagement in using ePATH as a self-management tool in the postoperative phase. ${ }^{11}$ On the other hand, the more autonomy, the more options there are to handle and the more digital literacy competence is required from both patients and healthcare professionals. Kim and $\mathrm{Xie}^{27}$ conclude that barriers to access online health information could depend on the availability and readability of content and the usability of e-Health services. To facilitate accessibility, educational programmes could be employed. Personalised information and tracking of symptoms and activities that can be deemed sensitive have to be protected 
by secure login, which might have a negative effect on user-friendliness and may challenge users' digital literacy. Thus, there is a delicate balance between supporting patient autonomy and individual expectations on what an e-Health application should offer. The usage of e-Health applications may also be affected by difficulties in fitting the technologies in daily life. ${ }^{28}$ Feelings of discomfort or lack of confidence in the technology may affect the usability of e-Health. Most technologies are designed for the general population and could pose a difficulty for groups that are unaccustomed to e-Health technology or have reduced cognitive, perceptual or psychomotor abilities. The acceptance of technology is fostered when it is perceived as non-intrusive or appealing, therefore careful considerations regarding the user interface are needed. ${ }^{28}$

\section{Fit between technology and task}

For a good fit between e-Health support and tasks, the tasks and processes need to be tailored and delivered in a manner that meets the user's expectations and needs. ${ }^{13}$ The technology, on the other hand, needs to have adequate functionality and stability to support the tasks. ePATH provided the patients with an easy way of getting in contact with healthcare, simplifying communication. Previous studies ${ }^{329}{ }^{30}$ have shown that patients in cancer care commonly felt abandoned after discharge from hospital. It was difficult to get in touch with the contact nurse or physician once at home, and worries and problems could pile up, with the patient not knowing where to turn. Another problematic task was keeping track of the large amount of information that patients received at the time of diagnosis. ePATH had adequate functionality to store diagnosis-specific information on treatment and rehabilitation, which was easy to access just-in-time when needed. Thus, the technology supported patients by providing information, as well as assisting in interaction and communication with healthcare in the postoperative phase. Information, interaction, visualisation and communication were identified as the cornerstones of the technology-task-fit in ePATH, as described previously. ${ }^{13}$

Patients' experiences of the technical functionality for supporting self-care tasks varied, as did patients' expectations of support and motivation to perform self-care activities. A computer-tailored intervention for physical activity (OncoActive) was found to increase physical activity in patients with prostate and colorectal cancer and survivors, but health-related effects on other measure were mainly found in those with colorectal cancer. ${ }^{7}$ In our study, it became clear that patients not only needed to be informed about what to do, but also needed to understand why they should perform self-care, and how to incorporate the new behaviours into their daily lives. In another study, ${ }^{26}$ avatars were suggested to function as a tutor or guide, to show and explain how to do certain exercises. An avatar could also be used represent the user and indicate their progress through the app. When someone is faced with a diagnosis and new behaviours of which they have little knowledge and experience, an autonomy-supporting healthcare climate with strong patient-staff or peer support is imperative to facilitate engagement in self-care activities. ${ }^{11}{ }^{19}$ In this context, an e-Health application may serve as a tool bridging distance and as continuous long-term support.

\section{Fit between the individual and the task}

For successful rehabilitation, patients need to be engaged and motivated to perform the recommended activities. ${ }^{24}$ In this study, the importance of diagnosis-specific information and self-care advice of personal relevance was appraised, showing a clear association with motivation to perform self-care activities. It was also important that the tasks were accompanied by instructions on how to track and interpret the registrations. However, motivation is complex. There are various types of motivation or behavioural regulation, broadly classified as autonomous self-regulation, controlled regulation and autonomous motivation. Autonomous motivation 'moves people to action,' driven by personal value and utility (e.g. personally wanting to maintain or regain good health). Autonomous self-regulation encompasses intrinsic and integrated motivation, which describes activities that are spontaneous or heuristic. Such activities give sufficient satisfaction to be sustained for their own sake; the engagement in an activity is congruent with central personal goals and values. PATH may have enhanced intrinsic motivation by providing patients with personalised information and encouraging them to decide autonomously when to interact and communicate with healthcare. Commercial health apps often use gamification to increase engagement and motivation. This could be in form of getting recognition from the app for finishing tasks or being able to acquire points to unlock new functionality. Another way of increasing engagement could be to enable users to connect with and compete with others. ${ }^{26}$ In contrast to externally prompted activities that are functionally dependent on rewards for their occurrence, autonomous motivation is strongly correlated with the maintenance of behavioural change over time, as well as with greater psychological health. However, controlled regulation, generally reflecting internal pressures such as contingent self-worth, guilt, shame and a need for approval, can also drive a specific behaviour for a short period of time. ${ }^{11} \mathrm{We}$ found that patients tended to do what they thought was important to get the healthcare staff's approval, instead of understanding the benefit of the activity for themselves. Such controlled behavioural regulation, in this case driven by approval from the contact nurses, has in other studies been beneficial in the internalisation of a new behaviour into more autonomous forms of selfregulation. ${ }^{19}$ If motivation is to be established, the patient needs to have functional ways to feel connected, which is one of the three pillars of motivation according to SDT. ${ }^{11}$ This study indicates that an e-Health application may be a useful tool for patients to become more independent and take on more responsibility for self-care activities over time. 


\section{Limitations}

This study has a number of shortcomings that must be considered when interpreting the results. The voluntary nature of participation in a sample of men who had the same diagnosis and had undergone the same treatment in a medium-sized hospital implies that participants represented a rather homogeneous group. Thus, the user experiences may not be representative for potential future users with other diagnoses and in other settings. The sample size of 15 patients could also be seen as too small. However, according to Albert, ${ }^{31}$ a study of eight to ten participants can provide meaningful results. The adequacy of the sample, the data quality and the variability of relevant experiences are often more important than the number of participants. We may have missed some difficulties in using the system features, as the usability tests were not video recorded. Log data covering frequency of actual use of different features in ePATH during the four test weeks are not included in this study. These limitations will be addressed further in evaluation of the application and user log data will be extracted in the ongoing effectiveness study to review actual use. ${ }^{20}$

\section{Conclusions and future research}

Advances in treatment have led to longer survival after prostate cancer diagnosis, but that does not automatically mean that survivors are living well. A substantial group of men surviving prostate cancer are experiencing longterm physical side effects and heightened psychological distress, indicating a need for long-term rehabilitation support and availability of healthcare. The patients' evaluation of ePATH as satisfactory, easy to use and o access, with information and activities tailored to patient needs and preferences, gives promising indications that e-Health applications are useful as support for self-management and rehabilitation after prostate cancer surgery. However, future development and research of ePATH need to include structured studies on functionality. Areas that were highlighted by users and functionality issues that may have passed unnoticed need to be highlighted in future work with the application. Further testing of ePATH regarding short-term and long-term effects on prostate cancer rehabilitation, health literacy, motivation and complexity of text is needed. The current study has provided information on development of additional features of relevance for the users. There is an ongoing Randomised Controlled Trial using ePATH,${ }^{20}$ in which a mobile application for rating symptoms is also available. However, elements of gamification, as suggested by the participants in the study of Jessen $e t a l,{ }^{26}$ are still to be developed. It has also been put forth that computer-based algorithms accounting for factors such as language, age, gender, reading ability and health literacy-level increase efficacy. ${ }^{32}$

As patients' needs for support varied across their rehabilitation trajectories, despite them having the same diagnosis and treatment, autonomous regulation and accessibility to healthcare seems crucial for the e-Health application to fit into their everyday lives.

Acknowledgements Thanks to the participating patients who took part in the study with the intention to improve ePATH. Thanks to Oscar Frykholm (OF) for valuable contributions to study design and data collection in the initial phase of the study.

Contributors LN responsibility: study design, acquisition of data, analysis, interpretation of data, drafting the article and intellectual content. AH responsibility: interpretation of data, drafting the article and intellectual content. CW responsibility: study design, acquisition of data, analysis, interpretation of data, drafting the article and intellectual content. ME responsibility: acquiring funding's, PI of the ePATH project, study design, analysis, interpretation of data, intellectual content and critical revision of the manuscript. KS responsibility: study design, acquisition of data, analysis, interpretation of data, drafting the article and intellectual content.

Funding This work was supported by the Cancer Foundation in Kalmar County, the Kamprad Family Foundation for Entrepreneurship Research and Charity, the Research Council of Southeast Sweden and the Swedish Cancer Society.

Competing interests None declared.

Patient consent for publication Not required.

Ethics approval The study was conducted in accordance with the ethical principles of the Declaration of Helsinki. Ethical approval was obtained from the Regional Ethical Board of Linköping University, Sweden, (Dnr 2016/484-31).

Provenance and peer review Not commissioned; externally peer reviewed.

Data availability statement All interviews and usability data are in Swedish and available on reasonable request.

Open access This is an open access article distributed in accordance with the Creative Commons Attribution Non Commercial (CC BY-NC 4.0) license, which permits others to distribute, remix, adapt, build upon this work non-commercially, and license their derivative works on different terms, provided the original work is properly cited, appropriate credit is given, any changes made indicated, and the use is non-commercial. See: http://creativecommons.org/licenses/by-nc/4.0/.

\section{ORCID iDs}

Camilla Wennerberg http://orcid.org/0000-0002-7158-2883

Mirjam Ekstedt http://orcid.org/0000-0002-4108-391X

Kristina Schildmeijer http://orcid.org/0000-0002-0895-674X

\section{REFERENCES}

1 Wilkens J, Thulesius H, Schmidt I, et al. The 2015 National cancer program in Sweden: introducing standardized care pathways in a decentralized system. Health Policy 2016;120:1378-82.

2 de Leeuw J, Larsson M. Nurse-Led follow-up care for cancer patients: what is known and what is needed. Support Care Cancer 2013;21:2643-9.

3 Schildmeijer K, Frykholm O, Kneck Åsa, et al. Not a straight LinePatients' experiences of prostate cancer and their journey through the healthcare system. Cancer Nurs 2019;42:E36-43.

4 Crawford-Williams F, March S, Goodwin BC, et al. Interventions for prostate cancer survivorship: a systematic review of reviews. Psychooncology 2018;27:2339-48.

5 Davis SN, O'Malley DM, Bator A, et al. Rationale and design of extended cancer education for longer term survivors (EXCELS) a randomized control trial of 'high touch' vs. 'high tech' cancer survivorship self-management tools in primary care. BMC Cancer 2019;19:340.

6 Frankland J, Brodie H, Cooke D, et al. Follow-Up care after treatment for prostate cancer: evaluation of a supported self-management and remote surveillance programme. BMC Cancer 2019;19:368.

7 Golsteijn RHJ, Bolman C, Volders E, et al. Short-Term efficacy of a computer-tailored physical activity intervention for prostate and colorectal cancer patients and survivors: a randomized controlled trial. Int J Behav Nutr Phys Act 2018;15:106.

8 Ruland CM, Maffei RM, Børøsund E, et al. Evaluation of different features of an eHealth application for personalized illness management support: cancer patients' use and appraisal of usefulness. Int J Med Inform 2013;82:593-603.

9 Bartlett YK, Selby DL, Newsham A, et al. Developing a useful, userfriendly website for cancer patient follow-up: users' perspectives on ease of access and usefulness. Eur J Cancer Care 2012;21:747-57. 
10 Kessel KA, Vogel MM, Kessel C, et al. Mobile health in oncology: a patient survey about App-Assisted cancer care. JMIR Mhealth Uhealth 2017;5:e81

11 Ryan R, Deci E. Self-Determination theory: basic psycological needs in motivation, development and wellness. New York, London England: The Guildford Press, 2017.

12 Corbin J, Strauss A. Managing chronic illness at home: three lines of work. Qual Sociol 1985;8:224-47.

13 Ammenwerth E, Iller C, Mahler C. IT-adoption and the interaction of task, technology and individuals: a fit framework and a case study. BMC Med Inform Decis Mak 2006;6:3.

14 De Vito Dabbs A, Myers BA, Mc Curry KR, et al. User-centered design and interactive health technologies for patients. Comput Inform Nurs 2009;27:175-83.

15 Frykholm O, Flink M, Lindblad M, et al. User-centered design of integrated eHealth to improve patients' activation in transitional care. Int J Integr Care 2016;16:A338.

16 Schildmeijer K, Wannheden C, Nilsson L, et al. Developing an eHealth tool to support patient Empowerment at home. Stud Health Technol Inform 2018;247:925-9.

17 Coleman EA, Parry C, Chalmers S, et al. The care transitions intervention: results of a randomized controlled trial. Arch Intern Med 2006;166:1822-8.

18 Flink M, Ekstedt M. Planning for the Discharge, not for Patient SelfManagement at Home - An Observational and Interview Study of Hospital Discharge. Int J Integr Care 2017;17:1.

19 Ng JYY, Ntoumanis N, Thøgersen-Ntoumani C, et al. SelfDetermination theory applied to health contexts: a meta-analysis. Perspect Psychol Sci 2012;7:325-40.

20 Ekstedt M, Schildmeijer K, Wennerberg C, et al. Enhanced patient activation in cancer care transitions: protocol for a randomized controlled trial of a tailored electronic health intervention for men with prostate cancer. JMIR Res Protoc 2019;8:e11625.

21 Bangor A, Kortum PT, Miller JT. An empirical evaluation of the system usability scale. Int J Hum Comput Interact 2008;24:574-94.
22 Association World Medical. Wma Declaration of Helsinki - ethical principles for medical research involving human subjects. Available: https://www.wma.net/policies-post/wma-declaration-of-helsinkiethical-principles-for-medical-research-involving-human-subjects/ 2018 [Accessed Jun 2019].

23 Elo S, Kyngäs $\mathrm{H}$. The qualitative content analysis process. J Adv Nurs 2008;62:107-15

24 Lentferink AJ, Oldenhuis HK, de Groot M, et al. Key components in eHealth interventions combining self-tracking and persuasive eCoaching to promote a healthier lifestyle: a scoping review. J Med Internet Res 2017;19:e277.

25 Page KL, Robson MJ, Uncles MD. Perceptions of web knowledge and usability: when sex and experience matter. Int J Hum Comput Stud 2012;70:907-19.

26 Jessen S, Mirkovic J, Ruland CM. Creating Gameful design in mHealth: a participatory Co-Design approach. JMIR Mhealth Uhealth 2018;6:e11579.

$27 \mathrm{Kim} \mathrm{H}$, Xie B. Health literacy in the eHealth era: a systematic review of the literature. Patient Educ Couns 2017;100:1073-82.

28 Bertoncello C, Colucci M, Baldovin T, et al. How does it work? factors involved in telemedicine home-interventions effectiveness: a review of reviews. PLoS One 2018;13:e0207332.

29 Lithner M, Klefsgard R, Johansson J, et al. The significance of information after discharge for colorectal cancer surgery-a qualitative study. BMC Nurs 2015;14:36

30 Mikkelsen TH, Søndergaard J, Jensen AB, et al. Cancer rehabilitation: psychosocial rehabilitation needs after discharge from hospital? Scand J Prim Health Care 2008;26:216-21.

31 Albert W. Measuring the user experience. Collecting, analyzing, and presenting usability metrics: Morgan Kaufmann Publishers Inc, 2013.

32 Jacobs RJ, Lou JQ, Ownby RL, et al. A systematic review of eHealth interventions to improve health literacy. Health Informatics $J$ 2016;22:81-98. 